\section{Propuesta para la reforma definitiva del sistema privado de salud chileno}

\author{
ALEJANDRA ZÚÑIGA F. ${ }^{a}$
}

\section{A proposal for the definitive reform of the private health insurance system in Chile}

Private health insurance should be able to provide coverage to people considered as high risk, such as women and the elderly. The only way to do that is to organize implicit or explicit cross-subsidies from low to high-risk individuals. This paper examines how European private health insurance companies introduced regulatory measures that could be introduced in Chile such as open enrollment, community-rated premiums, lifetime coverage, a package of minimum benefits and a risk equalization scheme.

(Rev Med Chile 2014; 142: 501-505)

Key words: Health benefit plans; Insurance, health; Managed care programs.

\author{
Universidad de Valparaíso, Chile y \\ Universidad Diego Portales. \\ ${ }^{a}$ Abogada. Doctora en Derecho. \\ Este artículo forma parte del \\ proyecto de investigación \\ FONDECYT No 1120022 \\ Chile. \\ Recibido el 15 de noviembre de \\ 2013, aceptado el 8 de abril de \\ 2014.
Correspondencia a: Alejandra Zúñiga Fajuri Errázuriz 2120. Valparaíso Teléfono: 32-250 7014 \\ alejandra.zuniga@uv.cl
}

E n la mayoría de los países miembros de la Comunidad Europea la demanda por seguros privados de salud ha ido aumentando año a año. Entre el período de 1987-1998, el gasto público en salud no ha podido solventar la demanda total de cuidado sanitario de la mayoría de los estados miembros ${ }^{1}$. La distribución de recursos asociados al derecho a la protección de la salud es también una preocupación primordial de la sociedad chilena, donde cada vez se ha ido imponiendo la idea de que el Estado debiera garantizar el acceso universal a cuidado sanitario apropiado en base a la "necesidad" de atención sanitaria, como sucede en Europa, y no en función del riesgo asociado o la capacidad de pago del enfermo, como era la tónica en los Estados Unidos de Norteamérica, antes de la reforma de Obama².

La reforma sanitaria chilena iniciada con el AUGE pretendió evaluar críticamente el funcionamiento de los seguros privados de salud (ISAPRES) desde el punto de vista de su capacidad para responder a las demandas de igualdad emanadas tanto por sus usuarios como por la reciente jurisprudencia del Tribunal Constitucional y la Corte Suprema. Sin embargo, los cambios que pudieron ver la luz en el sistema privado fueron pocos y muy insuficientes.
Las interrogantes que se han planteado son ${ }_{¿}$ Es legítimo permitir que las instituciones sanitarias, sean estas públicas o privadas, consideren criterios como la edad o el sexo del paciente para decidir sobre la adjudicación de bienes y servicios asociados al cuidado sanitario? ¿Pueden las ISAPRES asegurar su rentabilidad sin la utilización de estos factores, para los efectos de determinar la prima que deberá cubrir cada cual a la luz de su riego individual? Dar respuesta a estas preguntas, de la mano del análisis de la experiencia comparada, es el objetivo de las páginas que siguen.

\section{Los problemas del sistema privado de salud chileno}

Desde la sentencia 1.710 del año 2010 del Tribunal Constitucional, que declaró inconstitucional la clasificaciones de riesgo (actuarial insurance) de las aseguradoras privadas de salud, todos los actores sociales relacionados con el cuidado sanitario -legisladores, colegio médico, compañías de seguros, ONG's etc.- han intentado acordar una reforma legal capaz de superar el sistema vigente -desarrollado por los denominados "Chicago boys"- altamente segmentado y 
fuertemente inequitativo, por uno que, en la línea de los National Health Insurance (NHI) europeos, garantice cobertura sanitaria universal y acceso igualitario a cuidado sanitario básico, con una prima independiente del riego asociado.

El origen social y político de la reforma legislativa que se está discutiendo en la actualidad en el congreso (Boletín 8.105) determinará su viabilidad para alcanzar su objetivo -cual es, poner término a la judicialización del cuidado sanitario- en la medida en que sea capaz de crear una nueva institucionalidad para las ISAPRES que garantice, sin discriminación, el derecho a cuidado sanitario básico exigido por la justicia constitucional ${ }^{4}$.

Como ya hemos señalado en trabajos anteriores, nuestro país mantiene vigente un sistema sanitario mixto de consecuencias financieras fuertemente regresivas ${ }^{5}$ que se basa fundamentalmente en la segregación entre riesgosos y no riesgosos, con lo que se disponen primas muy diferentes por razón de sexo, edad y preexistencias. La actual avalancha de recursos de inaplicabilidad e inconstitucionalidad presentados ante el Tribunal Constitucional, así como los miles de Recursos de Protección que deben resolver las Cortes de Apelaciones del país (casi 70\% del total de recursos presentados en la actualidad son en contra de las ISAPRES) hacen urgente una respuesta del sistema que supere la discriminación arbitraria ${ }^{6}$.

\section{Mercados de seguros de salud privados (Voluntary Health Insurance-VHI)}

Puesto que el mercado de salud es un ejemplo clásico de asimetría de la información -tanto entre los usuarios y los administradores, como entre los usuarios y los proveedores- es necesaria la intervención del Estado para regular el sector y asegurar que el desbalance no se traduzca en resultados ineficientes e inequitativos, tales como la selección activa de personas con bajo riesgo de enfermedad, la segmentación de la población y la desviación de recursos hacia prácticas improductivas desde el punto de vista sanitario y económico.

Los mercados de seguros de salud sufren de ineficiencias provocadas por la naturaleza del riesgo sanitario, la información asimétrica entre aseguradores y consumidores y la ausencia de competencia perfecta. Como resultado, el seguro voluntario (privado) rara vez logra una adecuada cantidad y calidad de cobertura poblacional, fracaso ilustrado crudamente en los Estados Unidos de Norteamérica, donde uno de cada tres adultos menores de 65 años no tiene seguro médico o posee cobertura esporádica e insuficiente ${ }^{7}$. Realidad que se repite también en Chile en el sistema privado.

Por ello resulta interesante observar cómo en los Estados Unidos de Norteamérica y Chile, dos países con sistemas privados de salud libertarios y fuertemente desregulados, se ha alcanzado tal grado de malestar social, producto de las inequidades que generan, que las demandan por reformas profundas no se han hecho esperar ${ }^{8}$. En los Estados Unidos de Norteamérica, la reforma iniciada por el presidente Obama, que pretende garantizar un mínimo sanitario universal, ha comenzado a implementarse con enorme ansiedad por parte de la población. En Chile, esa reforma implica la transformación del sistema actual de seguros de salud en un sistema de seguridad social regido por los principios de universalidad, libre movilidad, no selección por ingreso, exclusiones por preexistencias o carencias y cobertura vitalicia ${ }^{9}$.

La demanda de equidad dirigida al sistema de ISAPRES parte de la base de que, según evidencia la experiencia comparada, las compañías aseguradoras privadas son capaces de obtener beneficios aun cuando se les prohíba determinar primas en base a la calificación de riesgo individual ${ }^{10}$. También es común la obligatoriedad del seguro sanitario como elemento característico de la seguridad social europea, así como la prohibición de cancelar pólizas o impedir su renovación basándose en el estado de salud de los cotizantes. La opción de obtener cobertura suplementaria o complementaria por sobre la cobertura básica inicial es bastante común y será, sin duda, la regla general en el caso de los afiliados a ISAPRES en Chile ${ }^{11}$.

\section{3. ¿Cómo funcionan los seguros privados en el mundo desarrollado?}

La mayoría de los estados miembros de la Unión Europea (UE) proporcionan cobertura pública universal o casi universal de cuidado sanitario como parte de un sistema más amplio de "protección social". Los seguros privados que ofrecen cobertura complementaria representan menos de 5\% del gasto total en salud, según la Organización para la Cooperación y el Desarrollo Económico. En algunos estados miembros, sin 
embargo, el seguro privado también contribuye a la protección social, proporcionando cobertura que sustituye o complementa el seguro obligatorio. Sin esta cobertura privada, que es adquirida por lo general por 10 a $20 \%$ de la población, la gente no estaría suficientemente protegida contra los riesgos financieros asociados a la mala salud ${ }^{12}$.

La "Tercera Directiva de Seguros no de Vida" (92/49/CEE) del Consejo de las Comunidades Europeas limita el alcance de la intervención gubernamental en los mercados privados de seguros de salud con algunas excepciones. La directiva incluye una disposición especial (artículo 54) sobre los Voluntary Health Insurance (VHI) que actúan total o parcialmente como sustitutos de la cobertura de los sistemas nacionales de seguridad social. Conforme a esta disposición, los estados miembros podrán adoptar disposiciones para proteger el "interés general" y el acceso universal a los seguros, con independencia de la edad o el estado de salud ${ }^{13}$.

La lógica subyacente en el artículo 54.1 es la de permitir la regulación del VHI que cumple una función de protección social al reemplazar al seguro público en la provisión del mínimo sanitario $^{14}$. Luego, si el seguro sustituye o complementa los servicios del sistema público, el gobierno puede exigir a las aseguradoras privadas: inscripción abierta, cobertura vitalicia, calificación comunitaria de las primas y un paquete mínimo estandarizado de prestaciones. Todo ello sumado a un fondo de compensación de riesgos ${ }^{12}$.

Los gobiernos de Alemania y los Países Bajos han utilizado el artículo 54.1 para justificar la intervención en los mercados privados donde la selección de riesgos impide que algunas personas mayores y con enfermedades crónicas alcancen un nivel de cobertura privada adecuada y asequible. Las medidas reglamentarias en ambos países (antes de 2006 en los Países Bajos) incluyen la provisión de cobertura vitalicia, beneficios mínimos estandarizados, precios garantizados y el establecimiento de subsidios cruzados directos o indirectos entre quienes tienen seguros privados y aquellos de cobertura legal obligatoria. El mercado irlandés también está estrechamente regulado, las aseguradoras deben ofrecer inscripción abierta y calificación comunitaria de las primas. Además, el Ministerio de Salud tiene la facultad de organizar un régimen de compensación de riesgos por un organismo regulador independiente ${ }^{15}$.

\section{Mecanismos que garantizan la equidad en el sistema privado de salud}

La única manera de hacer que el VHI sea asequible para las personas de alto riesgo de enfermedad es organizando, implícita o explícitamente, un sistema de subsidios cruzados entre los más riesgosos y los menos riesgosos ${ }^{16}$. Los seguros de salud privado en Europa garantizan la equidad en el acceso y la no discriminación en la cobertura del seguro en base a los siguientes mecanismos:

\section{i) Inscripción abierta (Open enrolment)}

La inscripción abierta impide la exclusión de personas al VHI en razón de sus pre-existencias o su mala salud. En otras palabras, garantiza cobertura sanitaria a todas las personas de una comunidad dada, pues las aseguradoras no pueden rechazar las solicitudes de afiliación por motivos de discapacidad o mala salud. Es un tipo de regulación clave para garantizar el acceso a cobertura sanitaria en la mayoría de los estados de la Unión Europea donde los seguros privados sustituyen, total o parcialmente, el seguro público. Es un requisito reglamentario para todas las aseguradoras en países como Irlanda desde 1994, Bélgica desde el año 2007, Alemania, desde 2009, Eslovenia, Hungría, Francia y Luxemburgo ${ }^{17}$.

\section{ii) Cobertura vitalicia (Lifetime cover)}

Especifica que los aseguradores no pueden negarse a renovar la cobertura, salvo circunstancias excepcionales ${ }^{18}$.

\section{iii) Calificación comunitaria de riesgos (Community-rated premiums)}

La calificación comunitaria, como base para el cálculo de la prima del VHI, es fundamentalmente diferente del método que se suele usar para determinar las primas de seguros, que es la clasificación por riesgo individual, donde las compañías de seguros calculan la prima a pagar por el asegurado potencial sobre la base de factores particulares como la composición familiar, edad, sexo, ocupación, estilo de vida (consumo de tabaco, peso, estado de salud, etc.). En un mercado comunitario de calificación el asegurador no puede calcular la prima sobre la base de factores de riesgo individual, sino más bien en función al riesgo que se aplica a todas las personas dentro del mercado en su conjunto ${ }^{19}$. En los Países Bajos y 
Alemania, por ejemplo, las calificaciones en base a la edad y el sexo de los asegurados son ilegales.

\section{iv) Paquete mínimo de servicios sanitarios garantizados (package of minimun benefits)}

Tal como lo ha hecho el AUGE en Chile (hoy, GES) las aseguradoras deben garantizar beneficios equivalentes a los definidos por el gobierno para el sistema público.

\section{v) Mecanismos de compensación de riesgos (risk equalisation schemes)}

Los esquemas de compensación de riesgos son una forma directa de intervención en el mercado de seguros, que implica transferencias financieras entre las aseguradoras con bajos riesgos hacia las aseguradoras con altos riesgos ${ }^{20}$. Son un elemento esencial de los mercados de seguros de salud con inscripción abierta y calificación comunitaria, donde se introducen para garantizar el acceso al seguro de salud y la competencia leal entre las aseguradoras ${ }^{21}$.

En fin, en Austria, Bélgica, Dinamarca, Finlandia, Francia, Alemania, Grecia, Irlanda, Italia, Luxemburgo, Países Bajos, Portugal, España, Suiza y Reino Unido se aplican todos o algunos de estos mecanismos para regular el sistema privado de salud y garantizar la equidad.

\section{Conclusiones}

Puesto que el objetivo prioritario en materia de política pública de salud es preservar el principio de atención sanitaria igualitaria y accesible a todos los ciudadanos en función de su necesidad y sin importar sus recursos o su riesgo personal, el seguro obligatorio, de alcance prácticamente universal, y con una provisión de servicios con alto grado de gasto público, es el estándar en los países desarrollados. Estas características han sido determinantes a la hora de entender la evolución de los mercados de VHI en Europa, sobretodo enfocado al tipo de servicios que el Estado no provee bajo el sistema público.

Los seguros sanitarios privados deben garantizar el acceso por medio del diseño de medidas capaces de proveer cobertura a las personas que pertenecen a grupos considerados de "alto riesgo", como son las mujeres y los ancianos. Una forma de promover la solidaridad de acceso sanitario es por medio de reformas y regulaciones que prohíben a las compañías o fondos de enfermedad rechazar posibles afiliados en base a su situación de salud. Estas regulaciones, para que sean exitosas, no deben ser sólo prohibitivas sino que deben, como se pretende en Chile, proponer la implementación de fórmulas de ajuste de riego y regulación de las primas, tal y cómo se ha propuesto en el Congreso con el Proyecto de Ley 8.105 cuya discusión se encuentra pendiente y que busca incorporar un Plan Garantizado de Salud en el sistema de seguros privados.

\section{Referencias}

1. Palm W. Voluntary Health Insurance and EU Insurance Directives: Between Solidarity and the market. En The Impact of EU Law on Health Care Systems. MacKee, Mossialos y Baten, eds. Bruxelles-New York; Wien: PIE-Peter Lang, 2002.

2. Zúñiga Fajuri A. When Constitutional Justice has the Last Word on healthcare. The case of Chile. Int J Health Serv 2014; 44(2): 373-81.

3. Zúñiga Fajuri A. El derecho a la vida y el derecho a la protección de la salud en la constitución: una relación necesaria. Revista del Centro de Estudios Constitucionales 2009; 9 (1): 37-64.

4. Zúñiga Fajuri A. Sistemas sanitarios y Reforma AUGE en Chile. Revista Acta Bioética 2007; 13 (2).

5. Zúñiga Fajuri A. Isapres, Tribunal Constitucional y distribución del derecho a cuidado sanitario. Rev Med Chile 2013; 141: 514-8.

6. Schoen C, Doty MM, Collins SR, Holmgren. A Insured but not protected: how many adults are underinsured? Health Affairs, Web Exclusive 2005; 14: W5289-W5-302.

7. Himmelstein DU, Thorne D, Woolhandler S. Medical Bankruptcy in the United States, 2007: Results of a National Study. Am J Med 2009; 8: 741-6.

8. Congreso Nacional de Chile. Proyecto que modifica el sistema privado de salud incorporando un plan garantizado. Boletín 2011; 8: 105-11.

9. Superintendencia de Salud de Chile. Propuestas de regulación al problema de los precios, planes y cautivos en Isapres y su Relación con el Fondo de compensación Solidario. Departamento de Estudios y Desarrollo, 2009.

10. Laske-Aldershof T, Schut E, Beck K, Gress S, Shmueli A, Van de Voorde C. Mobility in Social Health Insurance Markets: A Five-Country Comparison. Applied Health Econ Health Policy 2004; 3(4): 229-41. 
Propuesta de reforma del sistema de salud privado - A. Zúñiga et al

11. Thomson S, Mossialos E. Regulating private health insurance in the European Union: the implications of single market legislation and competition policy. Working Paper $\mathrm{N}^{\circ} 4$. The London School of Economics and Political Science, 2006.

12. Mossialos E, McKee M. EU Law and the Social Character of Health Care. Work \& Society 2002; 38. PIE-Peter Lang 2002; 259.

13. Thomson S, Mossialos E. Voluntary Health Insurance in the European Union. A critical assessment. Int J Health Serv 2002; 32 (1): 19-88.

14. Lewis R, Mays N. How to Regulate Health Care in England. An International Perspective. Londres, King's Fund, 2006.

15. Van De Ven WP, Schut FT. Guaranteed Access to affordable coverage in individual health insurance markets". The Oxford Handbook of Health Economics (Glied and
Smith Eds.), New York, Oxford University Press, 2011.

16. Thomson S, Mossialos E. Private health insurance in the European Union. Final report prepared for the European Commission. London School of Economics and Political Science, 2009.

17. Maynard A. The public-Private Mix for Health. The Nuffield Trust, Oxford, 2005.

18. Callahan D, Wasunna AA. Medicine and the Market. Equity v. Choice. The Johns Hopkins University Press, Maryland, 2006.

19. van de Ven WP, van Vliet RC. How can we prevent cream skimming in a competitive health insurance market? The great challenge for the 90s. Health economics worldwide. Amsterdam, Kluwer, 1992.

20. Puig-Juno, J. Managing risk selection incentives in health sector reforms. Int J Health Plan Manag 1999; 14: $287-$ 311. 